\title{
Método de análisis de necesidades de cursos de inglés con fines específicos a poblaciones de estudiantes de educación a distancia
}

Mónica Chacón*

Recibido: enero 2010 • Aceptado: marzo 2011

\begin{abstract}
RESUMEN
El presente artículo tiene como fin demostrar las razones que apoyan el uso del análisis de necesidades como primer paso en el diseño, planeamiento y aplicación de un curso de inglés con fines específicos. Para lograr este objetivo, es primordial definir en qué consiste el análisis de necesidades y cómo, cuándo y quién debe realizarlo. Además, se empleará como ejemplo la población actual de estudiantes del curso inglés instrumental para bibliotecólogos I, a la cual no se le aplicó el análisis de necesidades.
\end{abstract}

Palabras clave: Análisis de necesidades, enseñanza del inglés, inglés con fines específicos (IFE), contenidos temáticos, diseño de curso.

\begin{abstract}
The following article is aimed to show the reasons that support the usage of the needs analysis as the first step in the design, planning and delivery of an ESP course. In order to attain this objective, it is crucial to define what a needs analysis is and how, when, and who should carry it out. Besides, the current student population in the course "Technical English for Librarians" will be employed as case study since the needs analysis was omitted for this particular course.
\end{abstract}

Key words: Needs Analysis, English Teaching, English for Specific Purposes, Syllabus Course design.

* $\quad$ Máster en Inglés, Universidad de Costa Rica. Encargada de Cátedra de Inglés en la Universidad Estatal a Distancia, UNED. 


\section{Presentación}

El idioma inglés se ha convertido en un elemento más de la cotidianeidad. La manipulación del idioma se ha intercalado en el quehacer cotidiano debido a la expansión comercial y cultural. Sin importar la clase social o el nivel de escolaridad, el uso del idioma inglés está presente en las conversaciones escritas y orales. Por ejemplo, cada vez es más común leer y escuchar nombres de marcas, productos, lugares, y conceptos en inglés.

En el campo laboral, además, el equipo técnico, los recursos tecnológicos, y la mayoría de las fuentes bibliográficas, están en inglés. Asimismo, en el nivel educativo, la enseñanza del inglés se ha vuelto elemento esencial del currículo de la gran mayoría de las instituciones educativas. En otras palabras, el conocimiento del inglés se ha apoderado del quehacer cotidiano y el dominio de esta destreza es altamente apreciada en el nivel laboral.

Sin embargo, las necesidades lingüísticas no son las mismas para todos los aprendices. No solo se limita al nivel de dominio, la edad o etnicidad, sino también incluye el propósito o finalidad de formarse en el idioma. Esto significa que hay personas interesadas en dominar una destreza lingüística del idioma por razones de estudio o trabajo, así como en satisfacer una necesidad lingüística única para así completar su tarea educativa o laboral. Por tal motivo, es creada la rama de la enseñanza del inglés denominada IFE: inglés con fines específicos, la cual se dedica a crear programas de inglés para profesionales, en que los contenidos lingüísticos y temáticos están enfocados en los elementos requeridos para cumplir tareas propias de los ambientes laborales y educativos.

Frecuentemente, el proceso de diseño de los programas IFE omite un estudio analítico de las necesidades de la población meta. Dicho análisis de necesidades provee un panorama claro de las expectativas, tareas específicas y conocimientos previos de la población meta. Por ello, se realiza comúnmente antes de iniciar el diseño del curso de inglés con fines específicos, pero se repite durante el desarrollo del proceso de aprendizaje para obtener datos actualizados y encauzar el curso para satisfacer las necesidades. Otra ventaja del análisis de necesidades es que este representa un respaldo para el responsable del curso de inglés con fines específicos, debido a que el especialista cuenta con información de respaldo para las decisiones tomadas en relación con el curso.

Por otro lado, cuando el análisis de necesidades es omitido, el resultado del curso no es siempre exitoso, ya que las necesidades 
lingüísticas de los participantes no están presentes en las actividades. Además, se da como un supuesto que el inglés es imperante en el desarrollo profesional y académico de la población meta, y así se incluye en el aprendizaje formal e informal.

Con base en lo anterior, y la información recopilada en una encuesta realizada a estudiantes de inglés instrumental para bibliotecólogos I, se puede afirmar que el análisis de necesidades es un proceso crucial en el aprendizaje de un segundo idioma a nivel específico.

\section{Antecedentes}

El estudio e investigación en el campo del análisis de necesidades es parte de proyectos donde se diseñan e imparten cursos de inglés con fines específicos. Es decir, que la importancia de llevar a cabo un análisis de necesidades en un grupo meta es parte de un procedimiento, y como tal no debería ser omitido. Por ello, las experiencias que han sido publicadas, y que son utilizadas como modelos de enseñanza del inglés, incluyen en su proceso este análisis.

Hadermann y Rojas (2007) ci$\tan$ que:

"la enseñanza del Inglés - como segunda lengua -comenzó en la primera mitad del siglo 20 , en la universidad de Londres con los renombrados profesores Harold
Palmer, especialista en Fonética y Daniel Jones, lingüista teórico y aplicado (55)."

Desde ahí, se han presentado casos positivos en donde el análisis de necesidades guio el trabajo de los especialistas de idioma. Un ejemplo de esto es el estudio que realizó Drew (1993) acerca de la efectividad de los cursos de inglés de negocios. También, Puig y Reyes (2001) describieron la experiencia de desarrollar un curso de inglés con fines específicos para profesionales de la salud, en donde el análisis de necesidades incluye como:

“...marco de referencia el enfoque histórico-cultural de L.S. Vigotsky, el cual permite comprender el aprendizaje como resultado de la actividad social de los hombres, y muy especialmente, como resultado de sus interacciones en el seno de determinado contexto social, histórico e institucional que condicionan los valores, objetivos y métodos de trabajo profesional fundamentados en una ética acorde con los intereses sociales más valiosos y propios de la concepción humanista, los cuales guían el trabajo de la Educación Médica Superior en nuestra sociedad socialista (269)."

Otro ejemplo acerca de la relevancia del análisis de necesidades lo manifiesta Robinson (2000) al decir que, mediante el análisis, los cursos tienen la oportunidad de poner a 
prueba y desarrollar innovaciones antes de la puesta en práctica.

Finalmente, en Europa expertos llevaron a cabo la investigación acerca de

"la posibilidad de desarrollar cursos de idioma en un sistema en que las tareas son divididas en 'porciones de unidades, cada una de las cuales corresponden a un componente de las necesidades del estudiante y que está relacionada con todas las otras porciones.'” (Hadermann y Rojas, 2007:56).

Por lo tanto, esta investigación demostró, una vez más, que por medio del análisis de necesidades, se responde a las incógnitas propias del diseño de cursos de inglés con fines específicos.

\section{Fuentes teóricas}

A lo largo de este apartado, se describirán dos conceptos claves presentes en la investigación realizada, así como la perspectiva en la que se basó este trabajo.

En primera instancia, es crucial definir el concepto de análisis de necesidades y describir la importancia que tiene el mismo en el aprendizaje de una lengua extranjera. Como su nombre lo indica, se trata de determinar las necesidades lingüísticas de la población específica que participará en un curso de inglés con fines específicos. El análisis se lleva a cabo por medio de cuestionarios o entrevistas a los futuros participantes del curso, donde se determinará, entre otras cosas, el nivel de dominio de idioma, las tareas laborales y académicas donde necesita utilizar inglés, los motivos por los cuales desea aprender inglés, y demás. Estos instrumentos son aplicados por expertos en la enseñanza del inglés con fines específicos antes del diseño y planeamiento del curso.

Las herramientas brindan un panorama dividido en dos grandes áreas: lo que los estudiantes necesitan adquirir del idioma inglés para llevar a cabo sus funciones profesionales o académicas y lo que los estudiantes conocen y son capaces de hacer en el momento del análisis. Robinson (2000) lo denomina análisis de la situación meta y análisis de la situación presente o actual. Por lo tanto, los resultados que arroje este análisis determinan en parte los contenidos, objetivos y destrezas lingüísticas que formarán parte del curso.

El análisis de necesidades, como se mencionó, evalúa diferentes ámbitos del desarrollo del curso. Las respuestas conseguidas determinarán "el nivel de motivación de los estudiantes, la selección de actividades, el uso de la lengua nativa de los estudiantes (L1) en la sala de clases y las formas de enfrentar la cultura subyacente a 
cada lengua (L1 y L2)" (Villalobos, 2008). En otras palabras, el análisis de necesidades permite predecir cuál será la actitud del estudiante en el curso de inglés y cómo adecuar el aprendizaje para satisfacer las expectativas expuestas.

Otra razón para llevar a cabo el análisis de necesidades es para determinar el tipo de material y la destreza o destrezas lingüísticas que se desarrollarán en el curso. Dicho conocimiento se consigue por medio del análisis de las respuestas obtenidas con los instrumentos. Esta característica de los cursos específicos (contenidos y materiales exclusivos) hace que el valor de los mismos sea bastante elevado. Como consecuencia, es común la participación de un patrocinador (Robinson, 2000).

Se entiende por patrocinador la institución o persona responsable de cubrir los gastos del curso. Por ejemplo, puede ser el empleador de una compañía, una instancia del gobierno o, en el caso particular de la UNED, el patrocinador sería el encargado de programa. Es válido rescatar que el encargado de programa en la UNED no está pagando por el curso de inglés técnico; sin embargo, es el responsable de determinar las razones de incluir cursos de inglés dentro del programa.

La vinculación del patrocinador puede ser conflictiva en el momento de diseñar el curso, debido a que la visión del patrocinador puede distar de lo que los estudiantes expresan en los cuestionarios de análisis de necesidades. Sin embargo, el especialista en el idioma a cargo del diseño del curso debe buscar un punto intermedio e intentar satisfacer ambas partes.

Finalmente, la tabulación de la información recabada durante el análisis de necesidades y la toma de decisiones sobre el diseño del curso pueden tardar semanas o meses. La duración de este proceso se verá afectada por el número de participantes, lo extenso del instrumento de medición, las expectativas del patrocinador y lo detallado de los análisis. Por este motivo, en repetidas ocasiones los cursos de inglés con fines específicos o técnicos omiten el análisis de necesidades o reciclan un curso ya dado. Es decir, utilizan la misma información con otra población.

Con base en lo expuesto, se puede concluir que la omisión o reciclaje de un análisis de necesidades es nefasto para el aprendizaje. Sin estos resultados, el curso no responderá a las necesidades del grupo meta; el nivel de dificultad del idioma de los materiales y las actividades no estará acorde con los estudiantes. Además, la motivación de los estudiantes no será la misma, ya que su criterio no fue tomado en cuenta. 
A pesar las consecuencias negativas expuestas previamente que conlleva no realizar el análisis de necesidades, muchas instituciones y compañías brindan cursos de inglés con fines específicos sin antes realizar dicho análisis.

Un segundo concepto amplio y relevante en esta investigación es el de inglés con fines específicos (IFE $o$ en sus siglas en inglés ESP). En la UNED, estos cursos se conocen con el nombre de inglés técnico. Sin embargo, a pesar de que recibe múltiples nombres, todos poseen una característica en común: son dirigidos a una población específica que necesita el idioma inglés para llevar a cabo tareas propias de su función profesional. Esto significa que el idioma es el vehículo para completar las tareas laborales y no el centro del curso. Por ello, un curso IFE enfatiza ciertas destrezas lingüísticas: sólo las necesarias para poder trabajar.

Ya que estas nociones fueron aclaradas, es pertinente referirse al enfoque en que se desarrolló la investigación. Por la naturaleza de los cursos IFE, es ilusorio elegir un único enfoque porque excluirá aspectos relevantes que se pueden estudiar al utilizar otro. Por ello, se aplicó un enfoque holístico, conformado a partir de la lingüística y la pedagogía pertinente en la enseñanza del inglés con fines específicos. Las perspectivas empleadas permitieron conocer un panorama amplio de la efectividad del análisis de necesidades.

\section{Metodología y materiales}

La investigación se llevó a cabo con los estudiantes de la carrera de Bibliotecología, Bibliotecas Educativas y Centros de Recursos para el Aprendizaje (impartida en la UNED) matriculados en el curso de inglés instrumental para bibliotecólogos I.

Se escogió este curso y esta población porque en el tercer periodo del 2010 fue ofrecido por primera vez. Otra razón de peso es que las destrezas lingüísticas que forman parte de este requieren participación activa y asistencia a las tutorías de forma obligatoria. Este último aspecto contradice la filosofía de educación a distancia; sin embargo, por los objetivos del mismo es imperante que se realice así. Finalmente, este curso incluye un componente en línea: innovación en los cursos de inglés técnico de la UNED.

Este parte del nuevo plan de estudios de la carrera y es el primero de dos cursos de inglés con fines específicos, los cuales fueron establecidos por el encargado de programa y desarrollados por la cátedra de inglés técnico. Tanto los materiales, el contenido en bibliotecología, así como las destrezas 
lingüísticas, fueron elegidos por el encargado de la carrera. Lo anterior significa que el análisis de necesidades fue obviado en su totalidad, tal y como se comprobó al encuestar a los estudiantes en torno a si en algún momento fueron consultados con respecto a los cursos de inglés. El 100\% de los encuestados respondió que no, aunque aclararon que consideran importante adquirir el idioma.

Otro dato que enfatiza la ausencia del análisis de necesidades es el desconocimiento que existía sobre el nivel de idioma por parte de los estudiantes. Esto se evidenció en el primer encuentro de los especialistas con los estudiantes, debido a que las expectativas de los encargados eran muy altas y sorpresivamente los participantes poseían un nivel de idioma relativamente bajo.

Aparte de las entrevistas a los participantes, se utilizó el método de observación cualitativa. La observación se enfocó en la actitud y comentarios realizados por los participantes a lo largo de las interacciones personales en las tutorías, así como los comentarios electrónicos en la plataforma del curso. En estos intercambios se pudo evidenciar la ausencia de motivación intrínseca para el aprendizaje de la lengua y un desconocimiento de qué esperar del curso. En otros casos, los estudiantes tenían expectativas muy diferentes en torno al nivel de dificultad y los contenidos del curso.

Lo anterior es una situación común en la UNED, ya que todos los cursos de inglés técnico o con fines específicos han sido concebidos por el encargado de la carrera. Como se indicó, el encargado del programa es el patrocinador del curso, y en la educación a distancia, es el ente a cargo de decidir las materias y contenidos del programa, con ayuda de los especialistas pertinentes.

\section{Análisis}

La situación que se experimentó en el curso Inglés instrumental para bibliotecólogos I es universal en la población unediana porque ningún curso de inglés técnico realiza el apropiado análisis de necesidades. Es hasta cierto punto entendible que se omita dicho análisis debido a que la población que matriculará es desconocida y en cada período hay nuevos alumnos. Si se analizan periódicamente las necesidades, los cursos y los materiales, entonces estos podrían ser renovados constantemente. En consecuencia, se producen altos costos en preparación de materiales y personal; por ende, dicha parte del proceso es descartada. Sin embargo, una posible solución sobre la población meta del análisis de necesidades serían los graduandos de los diferentes programas de 
la UNED o profesionales de dicho campo. Estos ofrecerían información actual y precisa acerca de dónde, con qué frecuencia y para qué tareas requieren el idioma inglés.

Si se hubiese llevado a cabo el análisis de necesidades, se podría haber puesto en evidencia el nivel de dominio de idioma de los estudiantes. La realidad encontrada y observada en el curso fue que los participantes tienen poco o nada de conocimiento del inglés. Pero cuando se diseñó el curso se pensó para estudiantes de nivel intermedio. Como consecuencia de esta falta de información, el curso (su evaluación, actividades de las tutorías, materiales de trabajo, etc.) tuvo que ser ajustado sobre la marcha. Cabe mencionar que este tipo de situaciones podría pasar en cualquier curso a distancia, pero en los cursos de inglés con fines específicos se puede prevenir por medio del análisis de necesidades.

Una de las decisiones asumidas por falta del análisis de necesidades fueron los materiales y a la vez las destrezas que se desarrollarían en el curso. Los encargados de programas son expertos en su área de trabajo, y por supuesto conocedores de las tareas propias de la profesión. Sin embargo, la información recolectada por medio de un análisis de necesidades es crucial para determinar el tipo de material y las destrezas que se requieren en el campo laboral hoy. Además, el análisis de necesidades permite una visión objetiva de lo que se necesita, ya que un único punto de vista podría ser subjetivo.

Como aspecto final, se obviaron las necesidades de aprendizaje de los estudiantes. El análisis de necesidades, como se dijo, investiga las expectativas de los participantes: hace que el aprendizaje se vuelva personalizado. Sería ilusorio pensar que se diseñará un curso a la medida de cada estudiante, pero el aporte de los mismos facilita conocer los intereses y percepciones del aprendizaje de una segunda lengua.

\section{Conclusiones}

Por medio del contraste de esta investigación y de estudios en otros países, se puede concluir que el análisis de necesidades lleva al éxito de los cursos de inglés con fines específicos. En primer lugar, permite el involucramiento de los futuros participantes en la toma de decisiones sobre el aprendizaje, lo cual da como resultado el empoderamiento, una de las más reconocidas fuentes de motivación intrínseca. En segundo lugar, ayuda a los diseñadores del curso y patrocinadores a decidir los contenidos, destrezas lingüísticas y materiales óptimos para el cumplimiento de los objetivos de aprendizaje. 
Es imperante aclarar que la forma en que se han concebido y diseñado los cursos de inglés técnico responde a los recursos y políticas institucionales actuales, es decir, que los encargados de carrera o cátedra crean los cursos sin la ayuda del análisis de necesidades, debido a que la normativa de la UNED así lo estipula. Sin embargo, por medio de estudios como este se puede comprobar que el análisis de necesidades es una herramienta de enriquecimiento académico.

La UNED debe promover el análisis de necesidades en los cursos de inglés técnico. En una sociedad en que el dominio de una segunda lengua augura un mejor trabajo, es responsabilidad de las instituciones educativas fomentar las estrategias educativas para lograrlo. Por ello, la inversión de recursos en la actualización de contenido, materiales y enfoques pedagógicos de los cursos de inglés técnico por medio de un análisis de necesidades, es válida.

Es también oportuno considerar la realización de un cambio en la manera en que se han diseñado cursos hasta ahora, ya que existe evidencia que apoya la utilización del análisis de necesidades antes de la creación de cursos IFE.

\section{Bibliografía}

Hadermann, Carmen G.; Rojas, Ana M. (2007). Revisión de enfoques para enseñar idiomas. Revista Semestral de Humanidades y Educación, ago 2007, Vol. 2 Issue 3, p54-57.

Puig Espinosa, José Sergio; Reyes Figueroa, María de los Angeles. (2001). Una experiencia pedagógica en la enseñanza del inglés con fines específicos en la educación médica posgraduada. Revista Cubana de Educación Médica Superior, sep-dic 2001, Vol. 15 Issue 3, p268.

Robinson, Pauline. (2000). English for Specific Purposes. Routledge Encyclopedia of Language Teaching \& Learning, p196-198.

Stevens, Gillian; Jin, Kinam; Song, Hyun Jong. (2006). Short-term Migration and the Acquisition of a World Language. International Migration, 2006, Vol. 44 Issue 1, p167-180.

Villalobos Letelier, René. (2008). La enseñanza del Inglés como segunda lengua (ESL) y como lengua extranjera (EFL). Revista Semestral de Humanidades y Educación, dic 2008, Vol. 2 Issue 5/6, p179-182. 
